\title{
Design of hybrid electric heavy fuel MALE ISR UAV enabling technologies for military operations
}

\author{
Marco Fioriti \\ Department of Mechanical and Aerospace Engineering, Politecnico di Torino, Turin, Italy \\ Silvio Vaschetto \\ Department of Energy Engineering, Politecnico di Torino, Turin, Italy \\ Sabrina Corpino \\ Department of Mechanical and Aerospace Engineering, Politecnico di Torino, Turin, Italy, and \\ Giovanna Premoli \\ Leonardo S.p.A., Turin, Italy
}

\begin{abstract}
Purpose - This paper aims to present the main results achieved in the frame of the TIVANO national-funded project which may anticipate, in a stepped approach, the evolution and the design of the enabling technologies needed for a hybrid/electric medium altitude long endurance (MALE) unmanned aerial vehicle (UAV) to perform persistent intelligence surveillance reconnaissance (ISR) military operations.

Design/methodology/approach - Different architectures of hybrid-propulsion system are analyzed pointing out their operating modes to select the more suitable architecture for the reference aircraft. The selected architecture is further analyzed together with its electric power plant branch focusing on electric system architecture and the selected electric machine. A final comparison between the hybrid and standard propulsion is given at aircraft level.

Findings - The use of hybrid propulsion may lead to a reduction of the total aircraft mass and an increase in safety level. However, this result comes together with a reduced performance in climb phase.

Practical implications - This study can be used as a reference for similar studies and it provides a detailed description of propulsion operating modes, power management, electric system and machine architecture.

Originality/value - This study presents a novel application of hybrid propulsion focusing on a three tons class MALE UAV for ISR missions. It provides new operating modes of the propulsion system and a detailed electric architecture of its powertrain branch and machine. Some considerations on noise emissions and infra-red traceability of this propulsion, at aircraft level.
\end{abstract}

Keywords MALE UAV, Power management, Electric moto-generator, Electric system architecture, Hybrid propulsion, Multiphase electrical machine

Paper type Research paper

\section{Nomenclature}

\begin{tabular}{|c|c|}
\hline \multicolumn{2}{|c|}{ Definitions, Acronyms and Abbreviations } \\
\hline AAA & $=$ Anti-aircraft artillery; \\
\hline AoI & $=$ Area of interest; \\
\hline EM & = Electro-magnetic; \\
\hline $\mathrm{EO} / \mathrm{IR}$ & $=$ Electro-optic/infra-red; \\
\hline EPDS & $=$ Electric power distribution system; \\
\hline HALE & $=$ High altitude long endurance; \\
\hline ICE & $=$ Internal combustion engine; \\
\hline IR & $=$ Infra-red; \\
\hline ISR & = Intelligence surveillance reconnaissance; \\
\hline LIDAR & $=$ Light imaging detection and ranging; \\
\hline MALE & $=$ Medium altitude long endurance; \\
\hline
\end{tabular}

The current issue and full text archive of this journal is available on Emerald Insight at: https://www.emerald.com/insight/1748-8842.htm

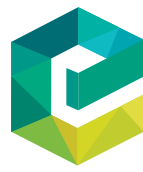

Aircraft Engineering and Aerospace Technology 92/5 (2020) 745-755

Emerald Publishing Limited [ISSN 1748-8842] [DOI 10.1108/AEAT-05-2019-0109]

$\begin{array}{ll}\text { ManPADS } & =\text { Man-portable air-defense system; } \\ \text { MTOM } & =\text { Maximum take-off mass; } \\ \text { UAV } & =\text { Unmanned aerial vehicle; } \\ \text { RF } & =\text { Radio frequency; } \\ \text { Rpm } & =\text { Round per minute; } \\ \text { SAR } & =\text { Synthetic aperture radar; } \\ \text { SFC } & =\text { Specific fuel consumption; and } \\ \text { SR SAMs } & =\text { Short-range surface-to-air-missiles. }\end{array}$

(C) Marco Fioriti, Silvio Vaschetto, Sabrina Corpino and Giovanna Premoli. Published by Emerald Publishing Limited. This article is published under the Creative Commons Attribution (CC BY 4.0) licence. Anyone may reproduce, distribute, translate and create derivative works of this article (for both commercial and non-commercial purposes), subject to full attribution to the original publication and authors. The full terms of this licence may be seen at http://creativecommons.org/licences/by/4.0/legalcode

This work has been developed in the frame of TIVANO Project (Tecnologie Innovative per Velivoli di Aviazione Generale di Nuova Generazione) supported by the Italian Aerospace Cluster funded by MIUR.

Received 31 May 2019

Revised 28 January 2020

Accepted 28 January 2020 


\section{Introduction}

In the ground of transportation sector, the hybrid-electric platforms are growing in number and application since the past decade because of many advantages, such as less fuel consumption and reduced environmental pollution to comply with more and more severe restrictions in terms of pollutant emission limits. To withstand these requirements, the conventional powertrains, which rely upon internal combustion engines (ICEs), have been increasingly improved reaching their technological limit. Considering the near future international regulations, ICEs could no longer withstand the more stringent pollution limits, especially in the automotive sector. This is leading to the adoption of the hybrid-electric propulsion system to increase the powertrain efficiency.

Hybrid-electric propulsion has been effectively implemented in automotive and naval transportation products in different ways and with different electrification levels. However, this technology is not often been considered for real aircraft applications (Zhang et al., 2008; Koster et al., 2011; Boggero et al., 2018) and the main reasons could be:

- The available technologies for energy storage lead to batteries/ultra-capacitors energy density relatively low compared to one of the liquid fuel. Therefore, the use of electric power could potentially increase the mass and complexity of the propulsion system.

- The resistance to use new equipment or systems that lack historical data of failure rate. This makes it difficult to predict system reliability and safety level.

Nevertheless, the hybrid/electric powertrain systems for aircraft are becoming interesting as aviation community is also pushing toward emissions reduction. The success and benefits of hybrid propulsion system in other industry fields started the transfer in the aeronautical one. At the early stage, this technology transfer has been only applied to general aviation aircraft (Glassock et al., 2017; Boggero et al., 2019; Airbus E-Fan project, 2018) and unmanned aerial vehicle (UAV) of the same class (Lieh et al., 2011; Hung and Gonzalez, 2012) to cope with the current maturity of the enabler technologies, airworthiness regulations and procedures and infrastructures for electric/ hybrid aerial platforms. However, several important studies are involving regional transportation as well (Isikveren et al., 2015; Antcliff and Capristan, 2017). The specific power density and performance of electric motors and batteries, the thermal management, the high conductivity and insulation materials, the energy safety and efficiency, the power distribution, the sub-systems integration (Fioriti et al., 2017) and the advanced control of on-board power electronics are just some of the research area, which directly affect the hybrid propulsion technology. Therefore, the development of hybrid/electric aircraft propulsion is progressing in parallel with the evolution of enabling technologies in the field of electrical systems.

Focusing on UAV aircraft class, the current applications are mainly related to small UAVs (i.e. micro, mini and tactical UAVs) with the advantage of low acoustic and visual signatures, but limited flight loitering time. This last characteristic is particularly significant for UAVs that use (even partially) electric motor supplied by batteries during loiter phase because, an increase of endurance leads to a heavier battery that, in turns, leads to a heavier UAV.
This paper explores from the electrical power generation standpoint the enabling technologies for a hybrid medium altitude long endurance (MALE) UAV designed for high endurance, medium altitudes intelligence surveillance reconnaissance (ISR) mission profiles enabling operations from benign/permissive scenarios (allowing to operate without any constraints resulting from benign or neutral actors or elements, factors in the environment are complexity and competitiveness) to contested and denied scenarios (i.e. penetration in hostile territory in the presence of surface to air and air to air threats).

The paper is divided into three main sections. First, the reference MALE UAV is described together with the possible hybrid propulsion system architectures. In the second section, the selected hybrid propulsion system is described focusing on the electric architecture and electric machine characteristics. Finally, in the third section, the main results, at aircraft level, have been stated comparing the same aircraft with the standard propulsion system and the one with hybrid propulsion.

\section{Medium altitude long endurance unmanned aerial vehicle case study}

Missions, threats and design drivers

The MALE UAV design drivers have been captured by taking into the account:

- the potential operational context (war fighting, security, peace support and peacetime military engagement) and mission profiles, including potential threats; and

- the inherent low agility of the MALE UAV that implies, once detected, the inability to escape from the engagement by opponent air defense systems. In this case, the only possibility to survive is to operate outside the threat envelopes or to fly at altitudes above the threat ceiling when the residual threats are limited to small arms, anti-aircraft artillery (AAA), manportable air-defense system (ManPADS) and short-range surface-to-air-missiles.

From the various mission phases, the following assessments have been drawn:

- Take-off and landing phase: Assuming proper base protection by other means, the absence of radar threats near the airfield can be assumed. However, pop-up infrared (IR) threats such as ManPADS or other personal carried weapons may be present in the area up to a distance to engage a UAV.

- Transit phase (ingress/egress): The flight profile can be adapted to avoid known threats (e.g. by pre-planning route or transit at high altitude). In case avoidance is not possible, the mission has to be aborted. For unknown threats, the question may arise if they can be avoided by stealth-driven features and this refers mainly to the radio frequency (RF) and IR signatures. It has to be stated that the necessary signatures levels to avoid the detection by a ground based acquisition threats is not achievable with a typical MALE design which is optimized for long endurance (e.g. high wing aspect ratio). Therefore, the best combination of internal and external layout features can enable lower observability level and support the capability to be warned in time of such a threat (by either onboard sensors and/or co-operative systems), with the aim to initiate some measures which may vary from the 
threat avoidance maneuver to self-protection measures up to mission abort.

- Operations in area of interest (AoI): If the presence of a threat is known or if it has been detected, the MALE UAV has to fly outside of the threat's engagement envelope and perform only stand-off ISR tasks or to abort the mission. If threats are difficult to be detected (small arms, AAA and ManPADS) the basic flight profile must keep out of their engagement range.

Signatures reduction aims to avoid or at least to delay detection and to improve the effectiveness of all self-protection measures, which are based on features to simulate equivalent deception signatures. Among others, the most dangerous phases of the mission were identified during take-off, landing and those immediately before/after to identify the UAV design drivers:

- From the one hand, by combining two different power sources: optimal combination between ICE and electric motor to obtain the best persistence capability together with lower acoustic and thermal signatures.

- From the other hand, by combining internal and external aircraft layout characteristics to provide high persistence (e.g. high wing aspect ratio) and reduced RF signatures (radar cross section). Obviously, if medium- or long-range surface-to-air-missile systems exist in the AoI, air superiority will first have to be established by other means before proceeding with missions.

\section{Main platform features}

In the light of above scenarios, the main objectives of the MALE ISR UAV design were to maximize the persistence and to minimize the exposure times in the threat area through $\mathrm{RF} / \mathrm{IR}$ reduced signatures to avoid or delay detection. The reference $\mathrm{UAV}$ is a tail-less aircraft with single engine pusher propeller (Figure 1). The maximum take-off mass (MTOM) is in the class of $3,000 \mathrm{~kg}$, the operative altitude is between 3.000 and $10.700 \mathrm{~m}$ and a maximum operating speed of about $115 \mathrm{~m} / \mathrm{s}$.

The reference ICE powertrain is a European 12-cylinder diesel/JET-A, water-cooled, in V-configuration with $373 \mathrm{~kW}$ maximum take-off power (Figure 2). The heavy fuel solution for military applications have been adopted as bringing several advantages with respect to conventional gasoline engines such as fuel consumption, robustness and reliability, availability of fuel, high specific power and less dangerous to store and handle.

The basic mission payload including ISR sensors (electrooptic [EO]/IR and radar) and weapons up to $680 \mathrm{~kg}$ to be installed in fuselage, including the use of an internal bay designed to minimize electro-magnetic signatures and carry up to $340 \mathrm{~kg}$ class and/or external under-wing hard-points. Two mission scenarios have been defined:

1 Mission "ISR Clean": $24 \mathrm{~h}$ at medium-high altitudes, $460 \mathrm{~km}$ range, with $230 \mathrm{~kg}$ internal payload (sensors).

2 Mission “Armed ISR": Loiter with $680 \mathrm{~kg}$ internal/external payload range and total endurance as fall-out.

\section{Hybrid propulsion architectures}

Analyses of the reference UAV with a hybrid propulsion system have been performed by Leonardo Aircraft Division and Politecnico di Torino within the TIVANO (Italian acronym for Innovative Technologies for New generation General Aviation Aircrafts) Project, funded by the Italian Ministry of University and Research (MIUR) within the framework of the CLUSTER projects, which had the target to develop new technologies for general aviation aircrafts and unmanned aircraft systems.

In details, the project aimed at developing key technologies in the following areas:

- diesel-electric hybrid powertrains as competitive alternative to the traditional AvGas powered ICE;

- new low-cost composite materials (design and manufacturing technologies); and

- electric brake-by-wire systems.

The powertrain hybridization has been investigated to further reduce the fuel consumption because of zero emissions operating modes (i.e. when only the electrical system is engaged) and IR signature reduction during specific segments of the reference mission profiles. Additionally, the safety level could increase, thanks to the capability of the hybrid powertrain to assist the emergency glide (Boggero et al., 2019) in case of engine failure.

Concerning the aeronautical sector, the main hybrid-electric powertrain architectures are the series and the parallel. However, some studies involved the power-split architecture as well. Following a brief description of the different architectures.

\section{Series hybrid}

For series hybrid architecture, the propeller of the UAV is directly driven by electric motor only. As shown in Figure 3, the electric motor is mainly supplied by the electric generator connected to ICE.

The electric power generated is managed by power converter units that can supply the electric motor and/or store the electric power in batteries or in ultra-capacitors (Koster et al., 2011). During cruise phase, the electric motor is entirely supplied by

Figure 1 Aerial platform views and details

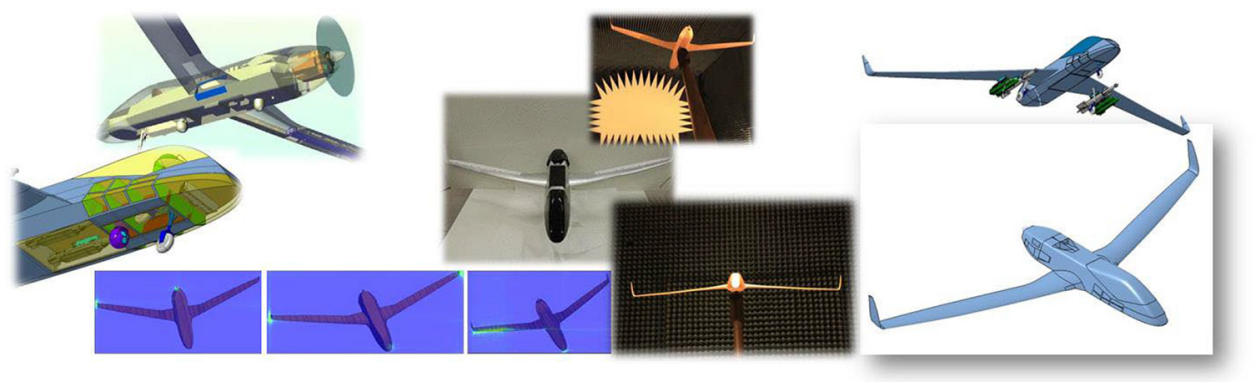


Figure 2 Diesel engine

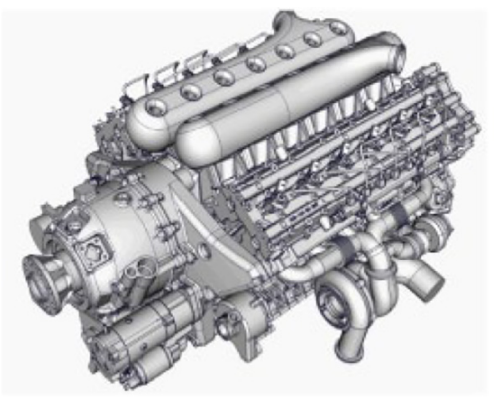

Figure 3 Layout configuration for the series hybrid architecture

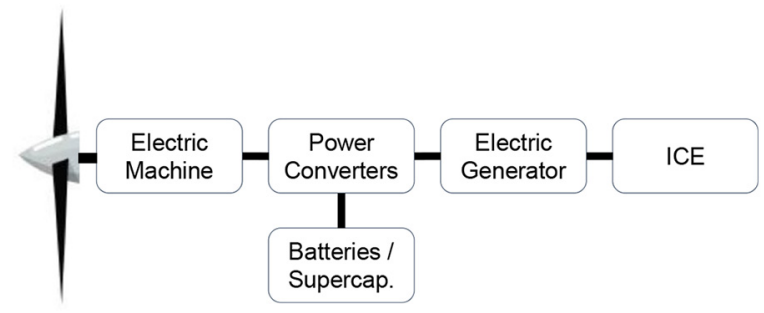

electric generator providing for a cruise endurance that is only limited by the fuel quantity. On the contrary, the batteries provide a temporarily additional power during takeoff, abrupt maneuvers and climb. During descent, the electric motor, that is typically a reversible electric machine, can generate power that may be stored in the batteries (or ultra-capacitors). One of the main advantages is the mechanical decoupling of the ICE from the propeller. In this way, the ICE can be set at its maximum efficiency working point as well as the propeller contributing to fuel burnt reduction. Moreover, the presence of the batteries that provide power for the more demanding mission phases allows the ICE downsizing. However, the reduction of ICE mass has to be balanced with the mass of additional equipment such as the electric machines, batteries and power converters.

\section{Parallel hybrid}

In parallel hybrid architecture, the ICE is coupled with an electric motor through a gearbox to drive the propeller, as depicted in Figure 4. As for serial hybrid, the electric motor is a reversible machine and can be used as a motor and as a generator. Therefore, when the electric machine is supplied with electrical power, it assists the ICE in driving the propeller

Figure 4 Layout configuration for the parallel hybrid architecture

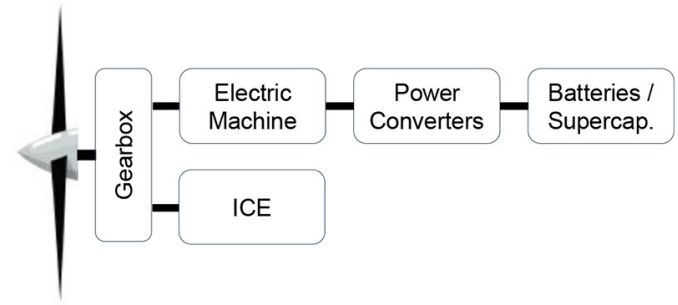

by means of a gearbox with a suitable gear ratio for propeller, ICE and electric motor that should rotate at different speed (expecially the electric motor in comparison with propeller and ICE).

When the electric motor is not electrically supplied, it is mechanically driven by means of gearbox and it acts as generator producing electric power that can be stored in the battery/ultra-capacitors.

Using parallel hybrid architectures, the ICE is not mechanically decoupled from the propeller and ICE cannot be always set at the best efficiency speed. However, the main advantage of this architecture is the ICE downsizing. Typically, the ICE has to provide only cruise power as maximum rating. The additional power necessary to complete take-off, climb and abrupt maneuvers is provided by the electric motor supplied by batteries. The downsized ICE is usually more efficient (i.e. smaller specific fuel consumption) in cruise phase reducing the amount of fuel required. The saved ICE mass could be totally wasted because of the mass of the additional equipment. However, compared to serial hybrid, the save in propulsion system mass is more achievable (Boggero et al., 2018). These, together with the fuel saved, may entail a reduction of the aircraft mass with a consequent mass saving.

\section{Power split hybrid}

The power split architecture uses two electric machines as depicted in Figure 5 to provide features of both serial and parallel hybrid (Olsen and Page, 2014). The ICE rotational speed is made decoupled from propeller speed by the epicyclical gearbox increasing ICE efficiency. With power split architecture, the ICE can provide both mechanical and electrical power as for parallel and series hybrid architecture, respectively. In this way, the overall efficiency of the propulsion system increases. However, the system complexity also increases adding more components than the other hybrid architectures. This leads to an increase of system mass and reduce its reliability. Therefore, this architecture is not further analyzed in this paper.

Comparison between series and parallel hybrid architectures

Parallel and series architectures have some common advantages, such as the ICE downsizing, as the ICE has to provide, at most, the power required for cruise in both the cases. The extra power, needed during takeoff and the other power demanding maneuvers, is provided by batteries or ultracapacitors by means of the electric motor. The parallel architecture could be considered a heavily modified state-ofthe-art propulsion system. The electric components manage a

Figure 5 Layout configuration for the power-split architecture

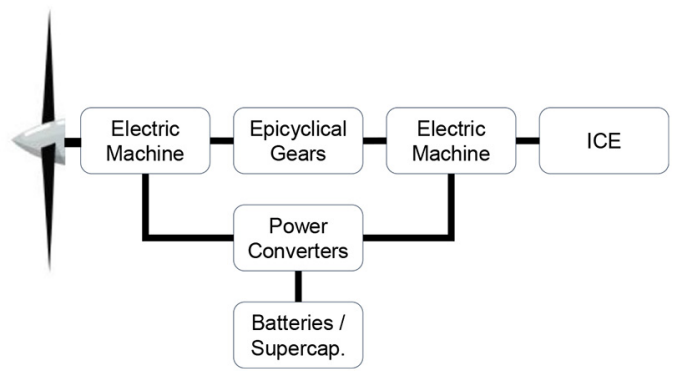


small percentage (in a range of 10-30 per cent) of the total propulsive power. The number of new components is limited as a possible estimated economic effort necessary to develop it. The series architecture has a very different approach. It could be considered a new propulsion system where the ICE is used to convert the chemical fuel energy into electrical energy. The electric components (except the batteries) manage the total propulsive power. In this sense, the serial hybrid is closer to allelectric propulsion system than the parallel hybrid. The drawback is the greater economic effort to develop it, the greater mass and quantity of the additional components that leads to an increase of total mass. Moreover, this architecture requires a double power conversion (i.e. mechanical-electricmechanical) reducing the total system efficiency. In the end, the series hybrid is usually more suitable for civil transport aircraft adding the gain of distributed propulsion than for light aircraft (Olsen and Page, 2014).

The two architectures present some similitudes in terms of energy management and control logic. In Figures 6-8, the different operative modes are depicted. In takeoff and climb maneuvers (Figure 6), both the architectures rely on the energy stored inside the batteries to supply the total power to the propeller. However, the ICE could be sized to provide up to the climb power. This design option is particularly beneficial when the hybrid propulsion system is installed in MALE or high altitude long endurance UAV. For these kinds of aircraft, the climb phase is very extensive over time and the use of batteries should be avoided to prevent an excessive increase of aircraft mass. In this specific case, the electric branch of the powertrain can be switched off for parallel architecture [Figure 6(d)] or the battery only for serial hybrid [Figure 6(c)].

During cruise [Figure $7(\mathrm{c})$ and (d)], the ICE provides the necessary power to sustain the flight. In some cases, the ICE has to also provide the power to recharge the batteries, totally or partially. This phase is depicted in Figure 7(a) and (b) for both hybrid architectures. During descent phase [Figure 8(a) and (b)], the aircraft potential energy can be recovered by means of propeller. In this case, the propeller is used in wind-milling regime (the propeller is rotated by aerodynamic forces) and it provides mechanical power to the electric machine recharging the batteries. The two architectures present some differences in this operating mode. For parallel hybrid, the ICE should be mechanically disconnected by means of clutch to allow the propeller to rotate. The series hybrid does not need this additional equipment as the ICE is already independent from the propeller. Moreover, the ICE can be turned on and contribute, if necessary, to the batteries recharge. The last operative mode provides for a total electric drive that is the rotation of the propeller using the electric power only [Figure 8(c) and (d)]. In both architectures, the electric power is provided by means of batteries. However, in serial hybrid only, it is possible to produce the total thrust as each electric component is sized for this. This is in contrast to the parallel hybrid that can only provide a partial extent of the total thrust.

The total electric drive operating mode can be used with different purposes. First, the taxi phase from hangar to runway and vice versa can be performed using only the electric branch to reduce ICE pollutant emission and usage and hence increasing time to overhaul. Second, in case of ICE failure, the electric motor would provide enough thrust to permit a controlled glide and landing. In particular, defining the safety altitude as the minimum altitude needed by the aircraft to return safely to land and to the airport after an ICE failure during takeoff maneuver, this operating mode reduces the safety altitude increasing aircraft safety (Boggero et al., 2019).

\section{Consideration on noise and infra-red emissions}

The aircraft noise and IR emissions are greatly affected by propulsion system (Moshkov and Samokhin, 2018; Rohacs et al., 2019). Therefore, a possible modification of the aircraft emissions can be supposed when a hybrid-propulsion systems is used. Focusing on noise emissions, it is important to consider

Figure 6 Power flow management during takeoff and climb for series and parallel hybrid architectures

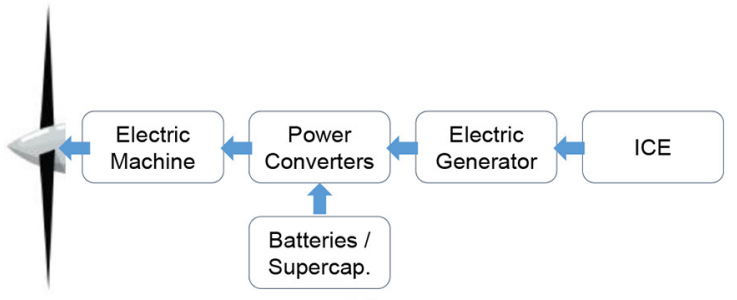

(a)

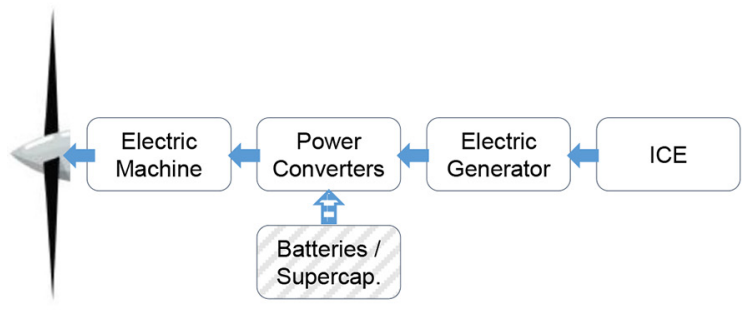

(c)

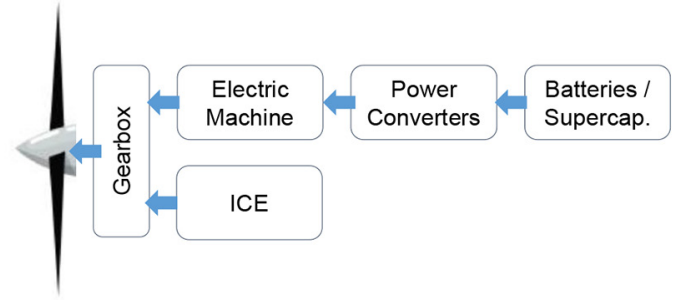

(b)

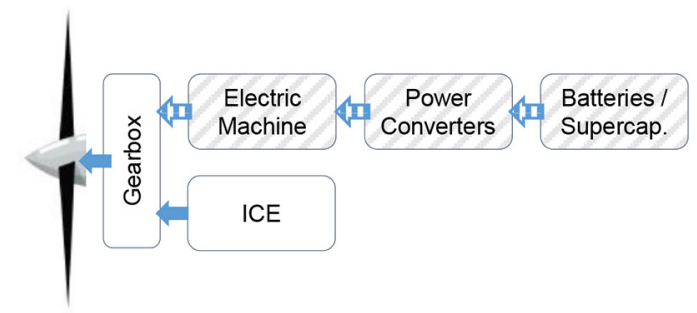

(d)

Notes: (a) Series hybrid - takeoff; (b) parallel hybrid - takeoff; (c) series hybrid - climb; and (d) parallel hybrid - climb 
Figure 7 Power flow management during cruise for serial and parallel hybrid architectures

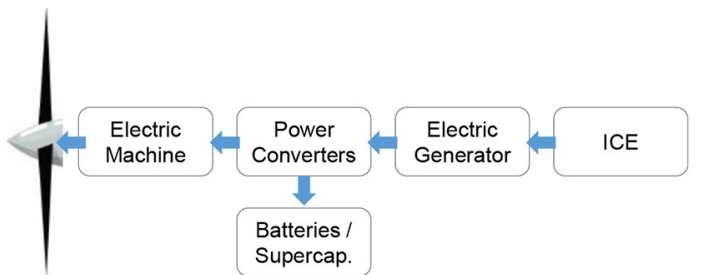

(a)

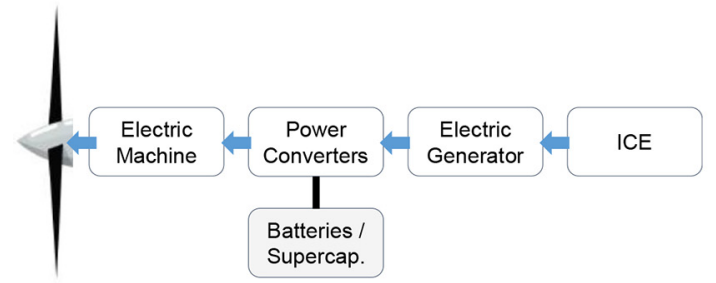

(c)

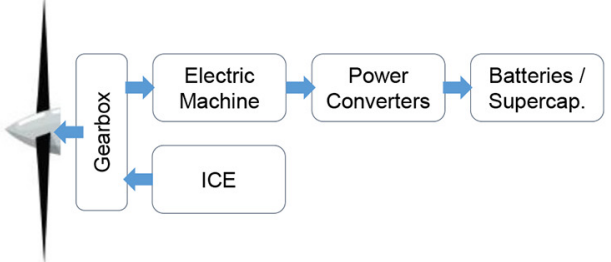

(b)

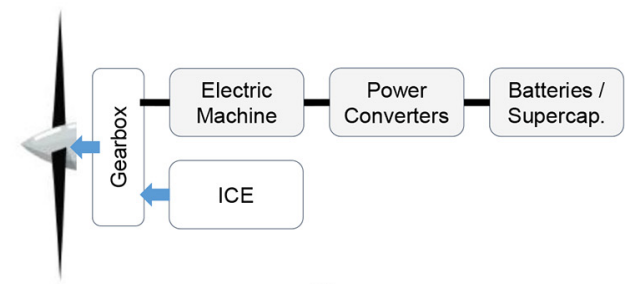

(d)

Notes: (a) Series hybrid - cruise with batteries charge; (b) parallel hybrid - cruise with batteries charge;

(c) series hybrid - cruise w/out batteries charge; (d) parallel hybrid - cruise w/out batteries charge

Figure 8 Power flow management during regenerative descent and taxi/emergency glide for serial and parallel hybrid architectures

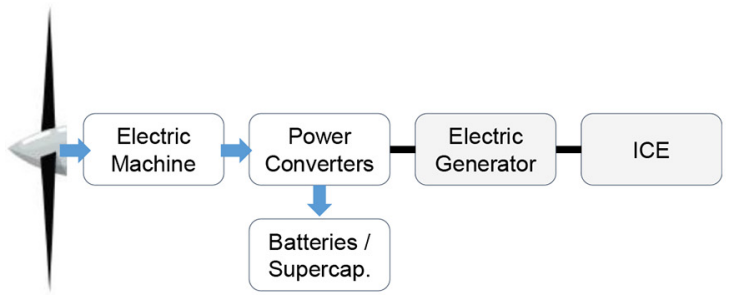

(a)

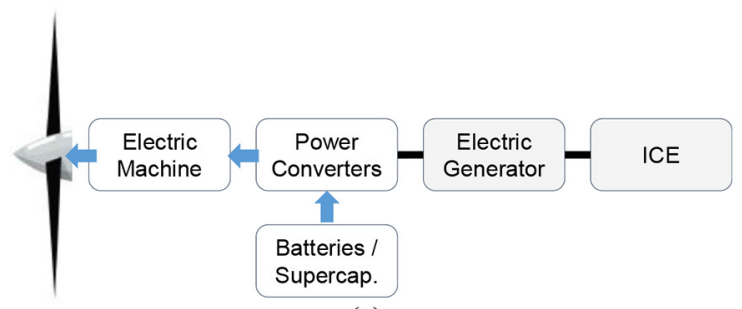

(c)

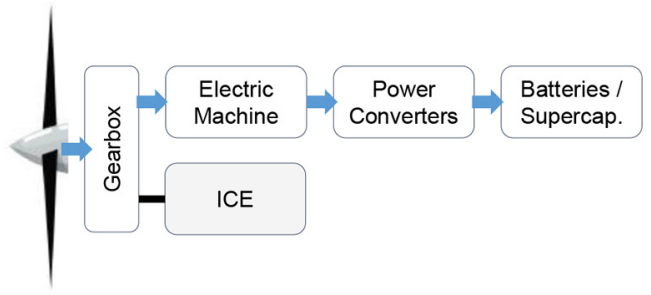

(b)

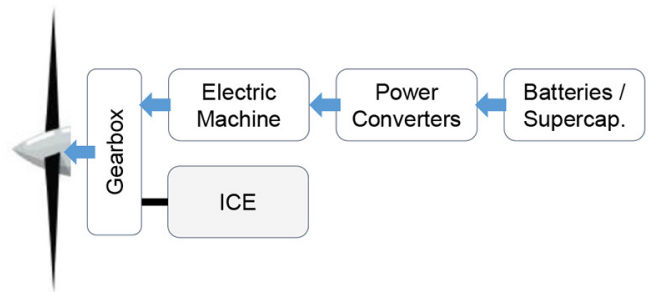

(d)

Notes: (a) Series hybrid - regenerative descent; (b) parallel hybrid - regenerative descent; (c) series hybrid - taxi/emergency glide; (d) parallel hybrid - taxi/emergency glide

that the propeller is the most significant noise source for a propeller-driven aircraft (Metzger, 1995). The noise produced by ICE is a secondary noise emission. Consequently, without any specific analysis, the proposed architectures of hybrid propulsion system seem not to be able to reduce significantly the aircraft noise emission. This is also true when they operate with the electric machine only.

In contrast, the IR emission could be modified by adopting the hybrid propulsion. First of all, the hybrid propulsion system generally being more efficient than standard one, the total amount of heat produced is slightly reduced. Secondly, when the system operates without any contribution from ICE (i.e. regenerative descent, taxi and emergency glide phases), the electric branch of the power plant produces a very low quantity of IR emissions preserving airplane controllability. However, more accurate analyses should be carried out to calculate the time necessary to cool down the hot parts of the ICE and to understand if this time could be compatible with an evasive maneuver.

\section{Hybrid propulsion system for reference medium altitude long endurance unmanned aerial vehicle}

Considering the lower impact on development cost, predicted reliability and, in general, on program risk, the parallel hybrid 
architecture has been selected to replace the conventional propulsion system of the reference MALE UAV. A more detailed architecture of the proposed propulsion system is depicted in Figure 9. The mechanical branch of the propulsion system consists of ICE with the necessary fuel tank and the clutch to disconnect it from the propeller. The electric machine, the propulsion batteries and some dedicated power conversion units composes the electrical branch (Airbus Press Release, 2017). The purpose of the converters will be discussed in the next section.

The main constraints that have guided the design of the hybrid powertrain are the following:

- the total mass of the hybrid configuration to not exceed the total value of the baseline without significant impacts on the aircraft balance;

- ensuring the performance required to fulfill the same mission profiles at the reference UAV;

- same external lay-out of the aircraft (in particular for engine nacelle);

- improve the minimum safety altitude during takeoff; and

- no impact on other aircraft systems (already designed as "more electric").

Considering mainly the mass requirement and the need to avoid any modification to the other sub-systems, the air cooling option has been selected to cool down the electric machine and the power converters. In this way, the mass and the complexity of the electrical branch is kept low. As with the low power density, the batteries are the only electrical equipment that could exceed the mass requirement. With the same iterative workflow proposed in Boggero et al. (2019) and keeping the

Figure 9 Main components of the hybrid-parallel architecture: mechanical (downward) and electrical (upward) branches

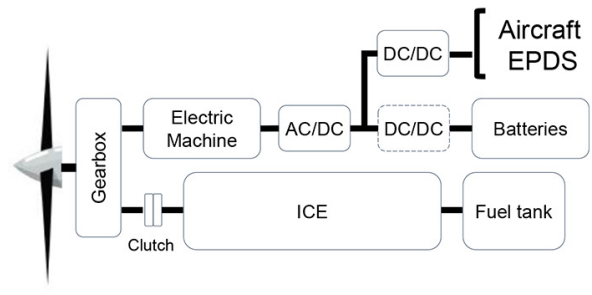

results of the conceptual design fixed (and aligned with case study UAV), the best hybridization degree is about 20 per cent. Therefore, ICE engine has been downsized (to reach around $300 \mathrm{~kW}$ of maximum power) in terms of number of cylinders in the hypothesis to maintain the same cylinder size to maximize its efficiency and to minimize its mass. Tables I and II list the amount of electric and mechanical power produced/required for different mission phases. In Table I, all mission phases where the electric machine is in motor mode are listed. In Table II the generator operation mode is presented.

Considering the entire propulsion system, the most demanding phase is take-off in which the maximum power is requested for both branches. When only the mechanical branch is considered and considering the effect of altitude, the inbound is another sizing phase. Focusing on the electrical branch, the controlled glide is another demanding phase also in terms of duration. This represents the most challenging requirement for batteries. However, for electric machine design, the coldcracking is the sizing phase. During this phase, the ICE has to start rotating at very low speed starting from $0 \mathrm{rpm}$ and it opposes a torque that is twice the one of the other power demanding phases. Therefore, the electric machine has to provide the highest torque at low rotational speed. This entails a relatively high current flow that sizes both the electric machine and related power converter. For this reason, the ICE starting by means of the main electric machine is optionally included in the design process. A dedicated electric starting system could be used instead of the main electric machine without any relevant effect on aircraft masses. In this specific case, the additional mass of the dedicated starting system is compensated by the mass reduction of the main electric machine. Moreover, as third option, the engine can be started taking advantage of the propeller inertia (the electric motor is used to rotate the propeller then the ICE is connected through the clutch and is started using the mechanical torque provided by the propeller itself.

Another important observation is the partial oversizing of the electric system when used to generate electric power. Looking at Tables I and II, it is evident that the power required by electric machine is less than the power provided by the same machine during the most demanding phases (i.e. takeoff and controlled glide). Considering also the partial overload of the electric machine during these phases, the machine can generate more power (almost double) of the requirement. Therefore,

Table I Power requirements for the electric machine in motor mode (1 p.u. max overall take-off power)

\begin{tabular}{lcccc}
\hline & Cold-cranking (optionally included) & Taxi & Take-off & Controlled glide \\
\hline Electric machine power & $0.01 \mathrm{p} . \mathrm{u}$ & $0.04 \mathrm{p} . \mathrm{u}$ & $0.2 \mathrm{p} . \mathrm{u}$ & $0.2 \mathrm{p} . \mathrm{u}$ \\
ICE power & $-0.01 \mathrm{p} . \mathrm{u}$ & $0 \mathrm{p} . \mathrm{u}$ & $0.8 \mathrm{p} . \mathrm{u}$ & $0 \mathrm{p} . \mathrm{u}$ \\
Time & $7 \mathrm{~s}$ & $240 \mathrm{~s}$ & $30 \mathrm{~s}$ & $80 \mathrm{~s}$ \\
\hline
\end{tabular}

Table II Power requirements for the electric machine in generator mode (1 p.u. max overall take-off power)

\begin{tabular}{lccc}
\hline & Climb & Inbound, outbound and cruise & Descent and landing \\
\hline Electric machine power & $0 \div 0.02 \mathrm{p.u}$ & $0.04 \mathrm{p} . \mathrm{u}$ & $0.02 \mathrm{p} . \mathrm{u}$ \\
ICE power & $0.07 \mathrm{p} . \mathrm{u}$ & $0.3 \div 0.5 \mathrm{p} . \mathrm{u}$ & $0.08 \mathrm{p} . \mathrm{u}$ \\
Time & $1.2 \mathrm{~h}$ & $22 \mathrm{~h}$ & $0.7 \mathrm{~h}$ \\
\hline
\end{tabular}


without any increase in mass, the electric system of this application of parallel hybrid propulsion is already compatible to any upgrade in ISR mission sensors or other powerconsuming equipment.

\section{Multi-phase electric system architecture}

The electric drive architecture, considered for the hybridparallel case of study, consists of a multiphase electric motorgenerator supplied by a voltage supply inverter (VSI) having a direct current (DC) link capacitor. The VSI power converter has been preferred to other solutions, such as matrix converters, for its lower complexity both in circuitry and control techniques.

Figures 10 and 11 sketch the two layouts that have been considered for the electric system. The two alternative schemes mainly differ on how the DC link is connected to the storage system. In particular, the layout in Figure 10 uses a bidirectional step up DC/DC converter to maintain the DC link voltage at $270 \mathrm{Vdc}$. This solution allows limiting the voltage at the storage system terminals in the range of $100-140 \mathrm{Vdc}$. On the other hand, the layout in Figure 11 directly connects the DC link at the storage system. In this case, to guarantee a DC link voltage approximately in the range of 200-270 Vdc, a higher number of electrochemical cells are required to achieve these voltage levels at the storage system terminals.

The preliminary trade-off analysis conducted for the case of study, mainly considered the overall system benefits in terms of increased safety and mass saving. In this perspective, the electric system layout that directly connects the DC link at the storage system (Figure 11) has been considered the preferred solution. Indeed, the removal of the DC/DC step up converter

Figure 10 Electric system layout with the DC/DC power converter for the bus voltage regulation

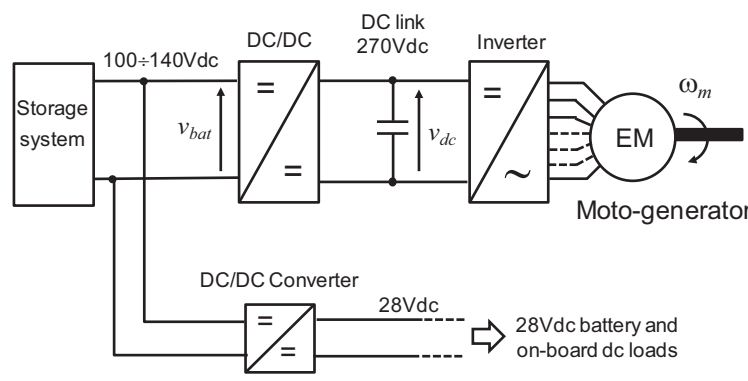

Figure 11 Electric system layout without the DC/DC power converter for the bus voltage regulation

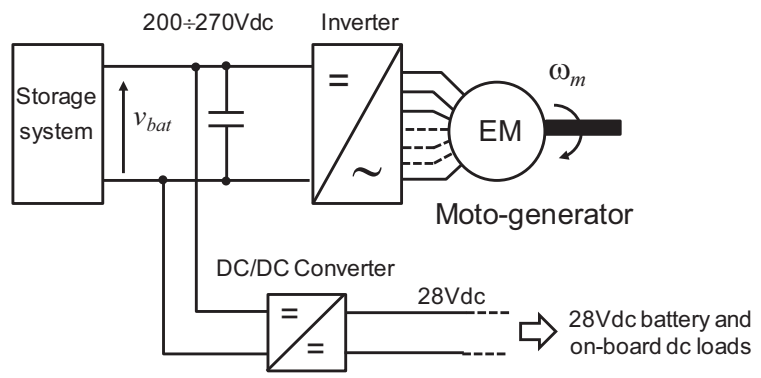

allows improving the safety because of the lower component count, as well as reducing the overall system mass. In particular, even if this solution requires a heavier storage system, it has to be considered that the air cooling requirement for the electrical components leads to non-negligible masses for power converters (Drofenik et al., 2011; Kolar et al., 2007).

For the electric motor-generator, a synchronous machine with permanent magnets has been considered as promising solution for this application, thanks to its higher torque density compared to other electric machine candidates (Ganev, 2014). During the operation in all the mission phases, the rotational speed of the electric machine is maintained almost constant in the range of 9,000-12,000 rpm by means of a mechanical gearbox. By the mission profile requirements listed in Tables I and II, the electric machine operates in steady state condition in generator mode. However, for the preliminary electromagnetic sizing of the motor-generator, an increased torque level has been properly considered to withstand the overload operations required in motoring mode to avoid the risk of demagnetization of the permanent magnets. Moreover, the motor-generator has been conceived considering tooth-wound coils. Indeed, compared to electric machines equipped with conventional distributed windings, the tooth-wound arrangements allow to achieve higher power and torque densities, thanks to their shorter end-winding connections. Also, it is widely recognized that these winding solutions increase the machine fault tolerance because of the improved electrical, thermal and magnetic separation among the stator phases.

To match the challenging fault-tolerant requirements imposed by the specific safety critical application, a multiphase drive configuration has to be used (Bojoi et al., 2019). A multiphase drive consists in having a number of phase higher than three both for the inverter and the electric machine. In this way, the electric drive features an inherent level of parallel redundancy as the motor-generator can be operated even in case of fault in one or more stator phases. Obviously, when one or more phases are missing, the healthy phases need to be overloaded to guarantee the same performance, if thermally admissible; otherwise, the electric machine can be operated at reduced functionalities.

Figure 12 shows the two multiphase configurations that have been considered for the parallel-hybrid case of study. In particular, the multiphase drive sketched in Figure 12(a) is based on multiple single-phase units having a dedicated power

Figure 12 Fault-tolerant multiphase drive configurations

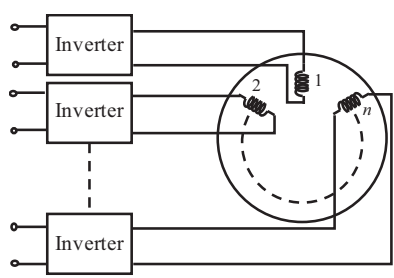

(a)

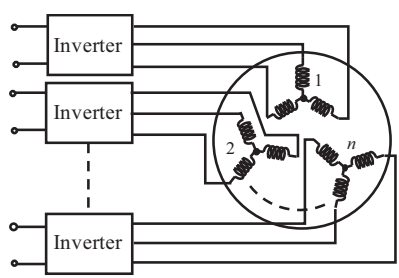

(b)
Notes: (a) Multiple single-phase units; (b) multiple three-phase units

Source: Bojoi et al. (2019) 
Figure 13 Comparison between three-phase and multi-phase electric drive solutions

\begin{tabular}{|c|c|c|c|c|}
\hline & & \multirow[b]{2}{*}{ Three-phase solution } & \multicolumn{2}{|c|}{ Multiphase solution } \\
\hline & & & $\begin{array}{l}\text { Multiple of independent } \\
\text { single-phase units }\end{array}$ & $\begin{array}{l}\text { Multiple of independent } \\
\text { three-phase units }\end{array}$ \\
\hline \multirow{2}{*}{ Inverter } & Mass & & & \\
\hline & Fault-tolerance & & & \\
\hline \multirow{2}{*}{ Electric motor-generator } & Mass & & & \\
\hline & Fault-tolerance & & & \\
\hline \multirow{2}{*}{ Overall system } & Mass & & & \\
\hline & Fault-tolerance & & & \\
\hline
\end{tabular}

converter to independently supply each stator phase of the electric motor-generator. In case of a fault, this solution allows disconnecting a single stator phase from the DC source; moreover, post-fault control strategies can be properly applied injecting independent current waveforms in the healthy stator phases to mitigate the fault effects. The multiphase drive sketched in Figure 12(b) is based on multiple three-phase units. In this case, each three-phase set is fed by a conventional threephase power converter. Besides the possibility of using the wellestablished three-phase power electronics modules, the multiple three-phase approach is usually based on simpler control schemes, thanks to the reduced number or independent currents compared to the multiple single-phase units. On the other hand, the multiple three-phase solution implies the disconnection of an entire three-phase set in case of a fault.

Figure 13 qualitatively compares the considered multi-phase electric drive solutions with the three-phase counterpart from the two main targets for the considered medium UAV MALE: the mass and the fault-tolerance features. While for the faulttolerance, the table summarizes the above evidence, for the mass it is possible to state that the multi-phase solutions slightly affect the machine mass because of the improved quality of the air gap magneto motive force compared to the three-phase counterpart. However, for the conducted preliminary analyses, the electric motor-generator presents an overall mass that is basically independent on the number of phases or three-phase sets, while the inverter and the overall aircraft system are marginally affected by the higher component count when multiple of independent single-phase units are used.

\section{"Electrification" main evidence}

Based on the preliminary design of the tail-less diesel MALE UAV and the following hybrid-electric feasibility study for the reference platform, the parallel hybrid architecture permits the downsizing of ICE compared to the traditional propulsion system designed for the same UAV.

Table III lists the main objectives achievable using the hybrid-electric powertrain. The figures are percentage with the baseline configuration as reference. The results are obtained using the ACAD tool (Fioriti, 2014) already calibrated on the reference aircraft with standard propulsion.

The ICE mass has been estimated starting from the engine cubic capacity and applying a linear proportionality. To consider the different scalability law of engine accessories, the total ICE mass obtained has been increased by 10 per cent. In this way, the ICE mass is reduced by about $20 \div 25$ per cent compared to ICE baseline. Considering the smaller usage range (the takeoff, climb and cruise regimes are essentially equivalent), the ICE thermodynamic cycle can be further
Table III Main objectives achievable by hybrid-electric powertrain having baseline configuration as reference

\begin{tabular}{llc}
\hline Mass variation & ICE mass & $-20 \div-25 \%$ \\
& Fuel mass for reference mission profile & $-10 \div-15 \%$ \\
& Propulsion system mass & $+25 \div+30 \%$ \\
& Take-off mass & $\approx-5 \%$ \\
Performance & Climb duration & $\approx+10 \%$ \\
variation & & \\
& Minimum safety altitude & $-45 \div-55 \%$ \\
\hline
\end{tabular}

optimized reducing engine SFC. The ICE downsizing and the increase efficiency in cruise entails a reduction of $10 \div 15$ per cent of the fuel burnt. The mass of the additional equipment of the hybrid propulsion systems is greater than the saved mass because of ICE downsizing. Hence, the mass of the hybrid propulsion system shows an increment of $25 \div 30$ per cent compared to baseline. This is mainly because of the batteries, which have a low energy density compared to kerosene. However, having the fuel saving a greater effect on the total aircraft mass, the MTOM shows a reduction of about 5 per cent. In terms of aircraft performance, the UAV using the hybrid propulsion is able to follow the entire mission profile except for the last extent of the climb. As shown in Table III, the total climb duration is increased by 10 per cent compared to baseline aircraft because of the downsized ICE. Against this drawback, the modified UAV enhances the performance in case of engine failure reducing the minimum safety altitude by half.

In Figure 14, the main advantages of the hybrid-electric powertrain have been summarized. The reduction of MTOM produces a consequent reduction of the thrust required during the whole mission. Moreover, the entire aircraft can be redesigned producing a further reduction of MTOM and required thrust. However, these additional advantages are not investigated in this analysis as the baseline UAV is considered fixed.

\section{Conclusion}

The implementation of parallel hybrid propulsion system on MALE UAV is an interesting test case because, for this class of aircraft, a relatively powerful electric machine is anyway necessary to supply the power demanding sensors such as EO/ IR, synthetic aperture radar, light imaging detection and ranging and satellite communication equipment. In parallel hybrid propulsion, this generator is used as electric motor by adding batteries, a gearbox and increasing the electric machine 
Figure 14 Main targets achieved with the hybrid-electric powertrain for reference case of study

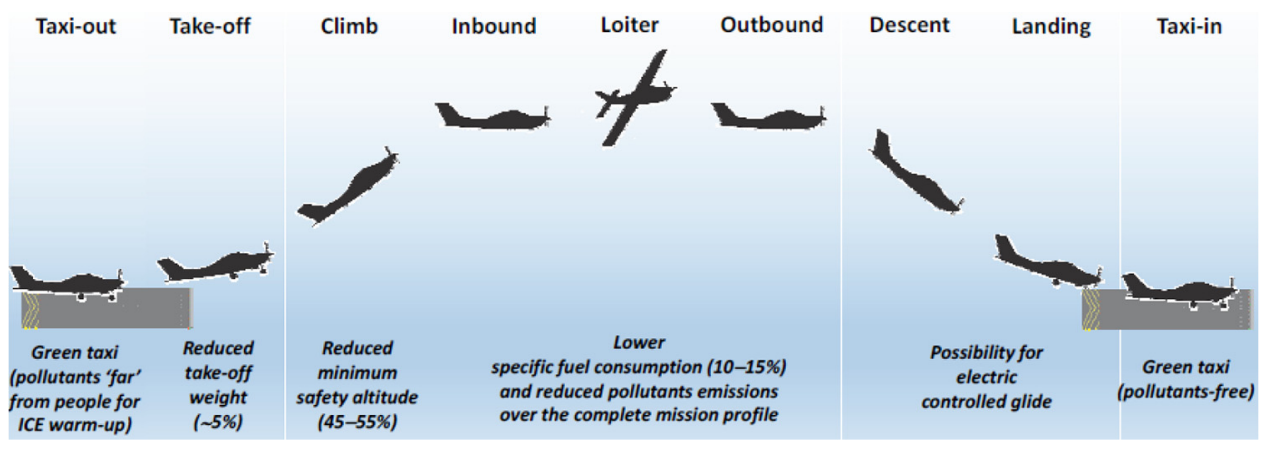

size. Therefore, using this specific architecture of hybrid electric propulsion system is possible to reduce the aircraft MTOM and the required fuel (or extend the mission range/ endurance). Another important advantage is the increase of aircraft safety level during take-off. Finally, by emission point of view, a downsized ICE intrinsically reduces its noise and IR emissions that can be further reduced, for a limited time extent, turning off the ICE and using the electric motor only.

As a general comment, UAVs represents an interesting case of study for the application of hybrid-electric powertrains. In fact, in their conventional configuration, these types of light aircrafts are usually equipped with "large" electrical generators to supply all the onboard sensors, imaging scanners and communication equipment. Therefore, during the flight phases where this equipment is not used, such as taxi and take-off, the electric machine can be operated as a motor for propulsion purposes.

The case of study highlights that the hybrid-electric solution leads to interesting advantages compared to the conventional powertrain based on the ICE. However, it is predictable that even larger benefits can be obtained if the aircraft' flight phases allows exploiting almost equally the electric machine as a motor and as a generator during the whole mission profile.

\section{References}

Antcliff, K.R. and Capristan, F.M. (2017), "Conceptual design of the parallel electric-gas architecture with synergistic utilization scheme (PEGASUS) concept", 18th AIAA/ ISSMO Multidisciplinary Analysis and Optimization Conference, p. 4001.

Airbus E-Fan project (2018), available at: www.airbus.com (accessed 16 December 2018).

Airbus Press Release (2017), "Airbus, Rolls-Royce, and Siemens team up for electric future partnership launches EFan X hybrid-electric flight demonstrator", available at: www.airbus.com (accessed 28 November 2017).

Boggero, L., Fioriti, M. and Corpino, S. (2019), "Development of a new design methodology for parallel hybrid aircraft", Proceedings of the Institution of Mechanical Engineers, Part G, F. Aerospace Engineering, ISSN 09544100.

Boggero, L., Fioriti, M., Ragusa, C.S. and Corpino, S. (2018), "Trade off studies of hybrid-electric aircraft by fuzzy logic methodology", International fournal of Applied
Electromagnetics and Mechanics, Vol. 56 No. S1, pp. S143-S152.

Bojoi, R., Rubino, S., Tenconi, A. and Vaschetto, S. (2019), "Multiphase electrical machine and drives: a viable solution for energy generation and transportation electrification", International Conference and Expo on Electrical and Power Engineering (EPE 2016), Iasi, 20-22 October, pp. 632-639.

Drofenik, U., Stupar, A. and Kolar, J.W. (2011), "Analysis of theoretical limits of forced-air cooling using advanced composite materials with high thermal conductivities", IEEE Transactions on Components, Packaging and Manufacturing Technology, Vol. 1 No. 4, pp. 528-535.

Fioriti, M. (2014), "Adaptable conceptual aircraft design model", Advances in Aircraft and Spacecraft Science, Vol. 1 No. 1, pp. 43-67.

Fioriti, M., Boggero, L., Corpino, S., Isyanov, A., Mirzoyan, A., Lombardi, R. and D'Ippolito, R. (2017), “Automated selection of the optimal on-board systems architecture within MDO collaborative environment", 18th AIAA/ISSMO Multidisciplinary Analysis and Optimization Conference, p. 31-50.

Ganev, E. (2014), "Selecting the best electric machine for electric-power generation systems: high-performance solutions for aerospace more electric architecures", IEEE Electrification Magazine, Vol. 2 No. 4, pp. 13-22.

Glassock, R., Galea, M., Williams, W. and Glesk, T. (2017), "Hybrid electric aircraft propulsion case study for skydiving mission", Aerospace 2017, Vol. 4, pp. 45.

Hung, J.Y. and Gonzalez, L.F. (2012), "On parallel hybridelectric propulsion system for unmanned aerial vehicles", Progress in Aerospace Sciences, Vol. 51, pp. 1-17.

Isikveren, A.T., Pornet, C., Vratny, P.C. and Schmidt, M. (2015), "Conceptual studies of future hybrid-electric regional aircraft”, Proceedings 22nd International Symposium Air Breathing Engines, Phoenix, AZ, pp. 25-30.

Kolar, J.W., Drofenik, U., Biela, J., Heldwein, M.L., Ertl, H., Friedli, T. and Round, S.D. (2007), "PWM converter power density barriers", Conf. Rec. IEEE-PCC, pp. 1-21.

Koster, J., Humbargar, C., Serani, E., Velazco, A., Hillery, D., Larrabee, D., Wormer, T., Marshman, J., Petersen, E. and Gaide, D. (2011), "Hybrid electric integrated optimized system (HELIOS) design of a hybrid propulsion system for aircraft", Conf. Rec. 49th AIAA, 4-7 January, pp. 1-10. 
Lieh, J., Spahr, E., Behbahani, A.e. and Hoying, J. (2011), “Design of hybrid propulsion systems for unmanned aerial vehicles", ALAA Foint Propulsion Conference E Exhibit, San Diego (CA).

Metzger, F.B. (1995), "An assessment of propeller aircraft noise reduction technology", NASA report, Langley Research Center.

Moshkov, P.A. and Samokhin, V.F. (2018), "Integral model of noise of an Engine-Propeller power plant", fournal of Engineering Physics and Thermophysics, Vol. 91 No. 2.

Olsen, J. and Page, J.R. (2014), "Hybrid powertrain for light aircraft", International fournal of Sustainable Aviation, Vol. 1 No. 1, pp. 85-102.
Rohacs, J., Jankovics, I., Gal, I., Bakunowicz, J., Mingione, G. and Carozza, A. (2019), "Small aircraft infrared radiation measurements supporting the engine airframe aero-thermal integration", Periodica Polytechnica Transportation Engineering, Vol. 47 No. 1, pp. 51-63.

Zhang, H., Saudemont, C., Robyns, B. and Petit, M. (2008), "Comparison of technical features between a more electric aircraft and a hybrid electric vehicle", Conference Rec IEEE VPPC, Harbin, 3-5 September, pp. 1-6.

\section{Corresponding author}

Marco Fioriti can be contacted at: marco.fioriti@polito.it 